\title{
Screening of the Salmonella paratyphi A CMCC 50973 strain outer membrane proteins for the identification of potential vaccine targets
}

\author{
TIAN-CI YANG ${ }^{2 *}$, XIAN-CANG MA ${ }^{3 *}$, FAN LIU $^{4 *}$, LI-RONG LIN ${ }^{2}$, LI-LI LIU ${ }^{2}$, \\ GUI-LI LIU ${ }^{2,4}$, MAN-LI TONG ${ }^{2,4}$, ZUO-GEN FU ${ }^{2,4}$ and LEI ZHOU ${ }^{1}$ \\ ${ }^{1}$ Department of Cardiology, The First Affiliated Hospital of Nanjing Medical University, Nanjing 210029; \\ ${ }^{2}$ Center of Clinical Laboratory, Zhongshan Hospital, Medical College of Xiamen University, Xiamen 361004; \\ ${ }^{3}$ Department of Psychiatry, First Affiliated Hospital of Medical College of Xi'an Jiaotong University, \\ Xian 710061; ${ }^{4}$ Medical College of Xiamen University, Xiamen 361004, P.R. China
}

Received May 19, 2011; Accepted September 9, 2011

DOI: $10.3892 / \mathrm{mmr} .2011 .587$

\begin{abstract}
Outer membrane protein antigens usually have strong immunogenicities, closely interact with the immune system and play a significant role in the development of new vaccines. The outer membrane proteins of Salmonella paratyphi $A$ (S. paratyphi $A$ ) were screened for immunogenicity and immunoprotection for potential vaccine targets. In this study, the bactericidal effect of antiserum against the total outer membrane proteins of S. paratyphi A CMCC 50973 strain was determined, and their immunoprotection was detected with a challenge experiment on vaccinated mice. The immunogenic outer membrane proteins were identified via immunoproteomic technology, and recombinant outer membrane proteins were expressed and purified. The immunoprotection provided by the immunogenic membrane proteins was verified through active and passive immunity challenge experiments. The result revealed a number of $S$. paratyphi $A$ outer membrane proteins that were proven as strong protective antigens. Twelve immunogenic outer membrane proteins were located and identified. Five recombinant proteins (LamB, pagC, TolC, $\mathrm{nmpC}$ and fadL) with strong immunoprotective abilities were found via the active immunity challenge experiment, with protection rates of $95,95,85,80$ and $70 \%$, respectively. They were also proven to induce good immunoprotection via the passive immunity challenge experiment, with protection rates of $65,55,60,55$ and $50 \%$, respectively. The immunoprotective
\end{abstract}

Correspondence to: Dr Lei Zhou, Department of Cardiology, The First Affiliated Hospital of Nanjing Medical University, Nanjing 210029, P.R. China

E-mail: zhoulei7005@hotmail.com

*Contributed equally

Key words: Salmonella paratyphi A, outer membrane protein, vaccine, immunogenicity, immunoprotection rate of the five-antiserum combination was $85 \%$. In conclusion, the LamB, pagC, TolC, nmpC and fadL outer membrane proteins, with strong immunogenicities and immunoprotection, are effective protein candidate targets for the development of new vaccines, whereas the recombinant outer membrane proteins are a promising tool for improving immunoprotection.

\section{Introduction}

The incidence of typhoid and paratyphoid fevers is increasing in Asia, particularly in the Indian subcontinent $(1,2)$. Clinical treatment has been difficult due to the widespread occurrence of clinically resistant and multidrug-resistant strains of Salmonella paratyphi A (S. paratyphi A) (3-5). Vaccination is one of the most crucial measures in preventing infectious diseases. The production and use of the traditional inactivated vaccine of $S$. paratyphi A were stopped due to the serious side effects of the vaccine. At present, the development of a safe and effective vaccine for paratyphoid fever A is urgent. Vaccines may be inactivated or attenuated microbial crude products, or a gradually developed subunit vaccine made from purified proteins, polysaccharides or nucleic acid (6-9). Proteins used as bacterial immunogens have many advantages, including safety, high conservation and strong immunogenicity. They also stimulate the $\mathrm{T}$ cell-dependent immune response. In the present study, immunoproteomic technology was used to study the outer membrane proteins of S.paratyphi A to screen for immunogenicity and protective immunity for use in the development of a new vaccine.

\section{Materials and methods}

Materials. The S. paratyphi A CMCC 50973 strain was obtained from the Chinese Medical Culture Collection (CMCC). DH-5a and BL-21 (DE3) competent cells were obtained from Tiangen Biotech Co. The pET-28a plasmid vector was conserved in our laboratory. The BALB/c mice were obtained from the Experimental Animal Center of the Xiamen University School of Medicine. 
S. paratyphi A outer membrane protein extraction. The outer membrane proteins of $S$. paratyphi $A$ were extracted using the method by Wooldridge and Williams (10). The content was detected using the Coomassie brilliant blue colorimetric method (ACTGene protein assay kit, USA). The extract concentration was adjusted to $2 \mathrm{mg} / \mathrm{ml}$ and stored at $-80^{\circ} \mathrm{C}$.

Preparation of immune serum from S. paratyphi A outer membrane proteins and determination of its in vitro sterilization capability. The BALB/c male mice (18-22 g) in the experimental group were intraperitoneally injected with $100 \mu \mathrm{g}$ outer membrane proteins, whereas $100 \mu \mathrm{l}$ normal saline was injected into the mice in the control group. Mouse serum from the experimental group was collected when the antibody titers in each mouse was $>1: 1,000$, as determined by the dotenzyme-linked immunosorbent assay (dot-ELISA) method. Up to $25 \mu 1$ newborn rabbit complement (Xiamen Boson Biotechnology Co., Ltd., China), mouse immune serum and CMCC 50973 (1,000 CFU/ml) were drawn up and co-cultured in tissue culture plates. The serum sterilization activity was detected by simulating in vivo conditions, using triphenyl tetrazolium chloride as the indicator.

Challenge experiment in mice vaccinated with S. paratyphi A outer membrane proteins

Determination of CMCC 50973 lethal dose $50\left(L D_{50}\right)$. The concentration of the CMCC 50973 suspension was $3 \times 10^{9} \mathrm{CFU} /$ $\mathrm{ml}$, as determined through the plate colony-counting method. Eight groups of BALB/c mice, each containing 10 mice, were injected with different doses of bacteria from $6.25 \times 10^{6}$ to $8 \times 10^{8}$. The number of deceased mice in each group was recorded following 7 days of observation. The results were plotted using the bacterial numbers as the abscissa and the mortality as the ordinate. Based on the experimental results, the series of dots were connected to form a curve, and a horizontal line was drawn from the $50 \%$ mortality point on the ordinate until it crossed the curve. A vertical line was then made from the intersection until it intersected the abscissa. The amount of bacteria corresponding to the point of intersection on the abscissa represented the $\mathrm{LD}_{50}$.

Mouse vaccination with the outer membrane proteins. The male BALB/c mice were divided into an experimental group and a control group, each containing 20 mice. The mice were vaccinated according to the aforementioned methods. The subsequent challenge experiments were conducted when the antibody titers of the mice in the experimental group reached 1:1,000. Otherwise, mouse immunization was strengthened accordingly.

Challenge experiments. Five times the $\mathrm{LD}_{50}$ of CMCC 50973 was injected into the experimental and control group mice. The number of deceased mice was recorded following 7 days of observation.

Screening and identification of the immunogenic outer membrane proteins using immunoproteomic technology. The screening and identification of the outer membrane proteins were conducted using two-dimensional gel electrophoresis (11). Three gels were prepared, two of which were stained with Coomassie brilliant blue G-250. The proteins in the remaining gel were transferred onto a polyvinylidine fluoride (PVDF) membrane and semi-dried, and the location of the immunogenic outer

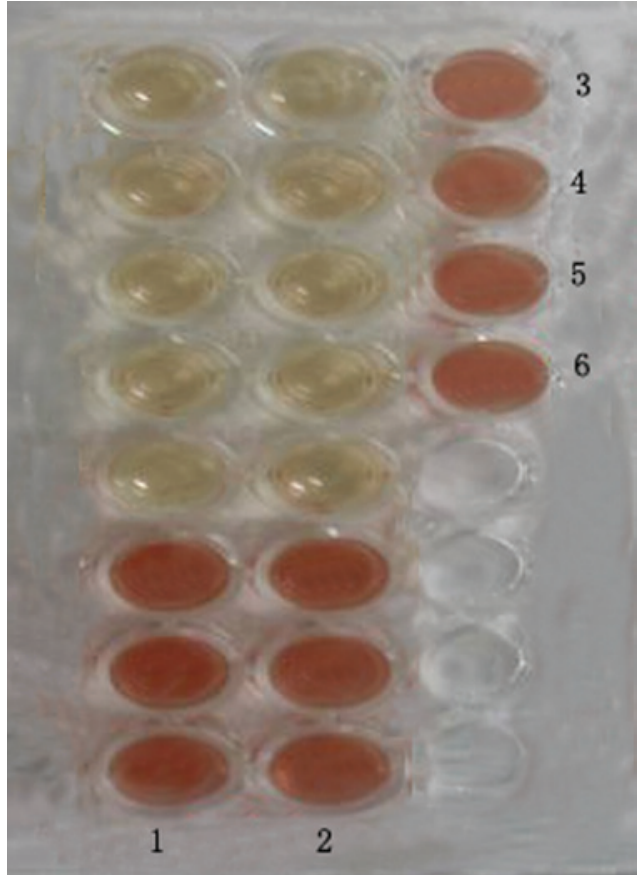

Figure 1. Bactericidal test results of the S. paratyphi A outer membrane protein antibodies. 1 and 2 , the outer membrane protein immune serum of CMCC 50973. 3 and 4, complement as a blank control to rule out interference from natural bactericidal substances. 5 and 6 , complement as an inactivated control, to confirm good bacterial activity.

membrane proteins was detected using Western blot analysis. Sera from paratyphoid fever A patients (with informed consent) were diluted 50-fold. The protein dots displayed on the PVDF membrane were compared to those on the two gels stained with Coomassie brilliant blue G-250. The corresponding protein dots were cut from the two stained gels. The peptide mass fingerprint (PMF) data of the enzymatically hydrolyzed proteins were obtained using a ReFlex ${ }^{\mathrm{TM}}$ III typed matrix-assisted laser desorption/ionization time-of-flight (MALDI-TOF) mass spectrometer (Bruker Co., Ltd., Germany). The protein identities were obtained by searching the Swiss-Prot database.

Prokaryotic expression and purification of immunogenic outer membrane proteins. The prokaryotic expression of the immunogenic outer membrane proteins was conducted according to the literature (11). The outer membrane proteins were purified using a Ni-NTA resin affinity chromatography column (Beijing Solarbio Science and Technology Co., Ltd., China), and processed according to the manufacturer's instructions.

Challenge experiment on the outer membrane proteinvaccinated mice (active immunity). Twenty mice were intraperitoneally injected with $100 \mu \mathrm{g}$ each of the purified immunogenic outer membrane proteins. When the antibody titers in the mice serum reached 1:1,000, the mice were injected with five times the $\mathrm{LD}_{50}$ of CMCC 50973. The challenge experiment was conducted on the vaccinated mice for 7 days to observe their morbidity and mortality rates.

Challenge experiment on the anti-recombinantoutermembrane protein antibody-vaccinated mice (passive immunity). Mice 
Table I. Results of the lethal dosage test of mice with CMCC 50973.

\begin{tabular}{lcccccccc}
\hline Group & 1 & 2 & 3 & 4 & 5 & 6 & 7 & 8 \\
\hline Bacterial content & $6.25 \times 10^{6}$ & $1.25 \times 10^{7}$ & $2.5 \times 10^{7}$ & $5 \times 10^{7}$ & $1 \times 10^{8}$ & $2 \times 10^{8}$ & $4 \times 10^{8}$ & $8 \times 10^{8}$ \\
Deaths & 0 & 2 & 3 & 5 & 7 & 7 & 9 & 10 \\
\hline
\end{tabular}

Table II. Results of the challenge experiment on mice vaccinated with the total outer membrane protein of $S$. paratyphi A.

\begin{tabular}{lccccccccccc}
\hline & $\begin{array}{c}\text { No. of experimental } \\
\text { animals }\end{array}$ & \multicolumn{4}{c}{ No. of deaths } & \multicolumn{2}{c}{$\begin{array}{c}\text { Total } \\
\text { deaths }\end{array}$} \\
\cline { 3 - 11 } & & 1st day & 2nd day & 3rd day & 4th day & 5th day & 6th day & 7th day & \\
\hline Experimental group & 20 & 0 & 0 & 0 & 2 & 0 & 1 & 0 & $3^{\text {a }}$ \\
Control group & 20 & 1 & 3 & 5 & 2 & 5 & 0 & 1 & 17 \\
\hline
\end{tabular}

${ }^{a}$ A significant difference between the experimental and control groups was observed using the Chi-square and Fisher's exact tests $(\mathrm{F}=19.6000$, $\mathrm{P}<0.05$; two-sided).

immunized with the outer membrane proteins were selected. When their antibody titers reached 1:1,000, blood was drawn to obtain the antiserum (the method is the same as method 2 of the outer membrane protein immune serum preparation).

Immunoprotection by single antiserum. Twenty mice from each group were vaccinated with single antiserum. The antiserum was injected into the caudal vein of the mice in the experimental group, whereas $200 \mu \mathrm{l}$ saline was injected into the control group. After $1 \mathrm{~h}$, the mice in both the experimental and control groups were injected with five times the $\mathrm{LD}_{50}$ of CMCC 50973. The morbidity and mortality rates of the mice were observed for 7 days.

Immunoprotection by mixed antisera. Equal volumes of several antisera were mixed. The experiment was repeated as previously described using the pooled serum instead of the single antiserum. The immunoprotection provided by the pooled antiserum was observed.

Statistical analysis. All statistical analyses were conducted using SPSS v13 for Windows. A Fisher's exact probability test was used to determine the significant differences across groups. A two-sided $\mathrm{P}<0.05$ was considered significant.

\section{Results}

In vitro bactericidal capability of serum from mice vaccinated with outer membrane proteins. The total anti-outer membrane protein mouse serum of $S$. paratyphi A was collected and co-incubated with newborn rabbit complement and S. paratyphi $A$ in the tissue culture plates. The results showed that the bactericidal antibody titer of the serum from mice vaccinated with the CMCC 50973 outer membrane proteins was 1:32 (Fig. 1).

CMCC $50973 L D_{50}$. The lethality test was conducted on BALB/c mice using different doses of CMCC 50973. The results showed that the mice injected with $8 \times 10^{8}$ bacteria all succumbed after 7 days, whereas the mice injected with $6.25 \times 10^{6}$ bacteria all survived. Different doses resulted in different mortality rates

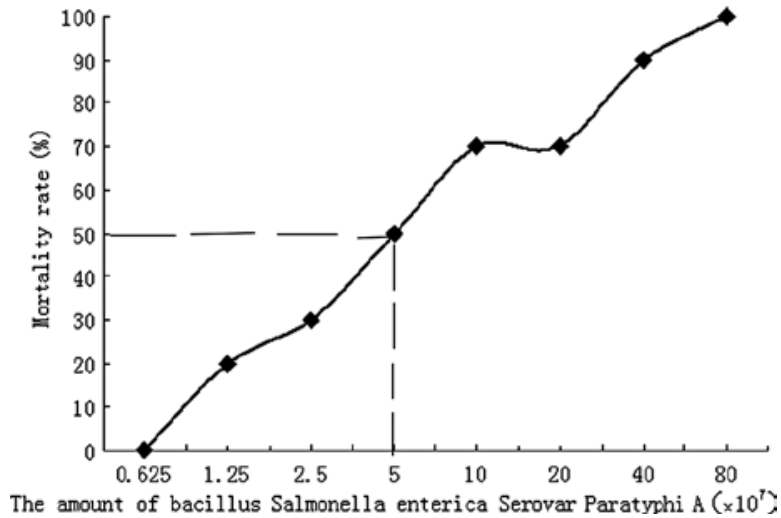

Figure 2. Lethality rate in mice with S. paratyphi A.

(Table I). A lethality curve was prepared based on the results, and a $5 \times 10^{7} \mathrm{LD}_{50}$ for CMCC 50973 was determined (Fig. 2).

Immunoprotection from total outer membrane protein. The $\mathrm{BALB} / \mathrm{c}$ mice were vaccinated with the total outer membrane protein of CMCC 50973. When the antibody titers reached 1:1,000, the mice were challenged with five times the $\mathrm{LD}_{50}$ of CMCC 50973. Up to 17 mice succumbed in the control group ( $85 \%$ protection rate). Two mice succumbed on the fourth day and 1 succumbed on the sixth day in the experimental group (Table II). A significant difference between the two groups was observed using the Chi-square and Fisher's exact tests ( $\mathrm{F}=19.6, \mathrm{P}<0.05$; two-sided).

Identification of immunogenic outer membrane proteins using immunoproteomic methods. Two-dimensional gel electrophoresis was used to analyze the CMCC 50973 outer membrane proteins. The serum from a paratyphoid fever A patient was used in the Western blot analysis (Fig. 3). A total of 37 spots were found in the transfer coating, with 19 corresponding to protein spots in the gel and the other 18 not corresponding to any spot because of low abundance. The 19 protein spots found were identified through mass spectrometry. Nine spots 


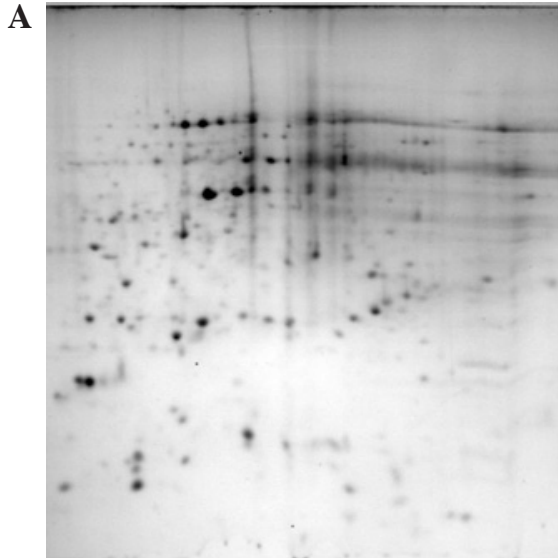

2-D PAGE

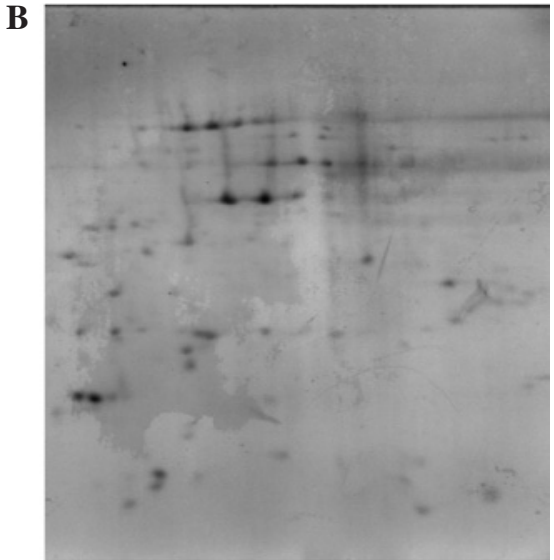

Western blotting

Figure 3. Results of the two-dimensional gel electrophoresis and Western blot analysis of the CMCC 50973 outer membrane proteins. (A) Two-dimensional gel electrophoresis of the CMCC 50973 outer membrane proteins. (B) Western blot analysis of the CMCC 50973 outer membrane proteins with the serum of a paratyphoid fever A patient.

Table III. Identification of the immunogenic outer membrane protein of CMCC 50973.

\begin{tabular}{rcccllr}
\hline No. & NCBI GI identifier & Mass (Da) & pI (calc) & \multicolumn{1}{c}{ Protein } & Gene & Score \\
\hline 1 & 56416031 & 50640 & 4.87 & Maltoporin precursor & LamB & 139 \\
2 & 56415127 & 553649 & 5.43 & Outer membrane channel precursor protein & tolC & 106 \\
3 & 56412835 & 41214 & 4.63 & Outer membrane protein C & ompC & 117 \\
4 & 56412712 & 47675 & 4.90 & Long-chain fatty acid transport protein precursor & fadL & 90 \\
5 & 56415967 & 68470 & 5.40 & Vitamin B12 receptor protein & btuB & 87 \\
6 & 56412364 & 89861 & 5.30 & Organic solvent tolerance protein & imp & 203 \\
7 & 56413481 & 39655 & 4.66 & New outer membrane protein: predicted bacterial porin & nmpC & 123 \\
8 & 56413763 & 20090 & 6.28 & Outer membrane invasion protein & pagC & 110 \\
9 & 56413343 & 22990 & 5.64 & Putative outer membrane protein & ompW & 96 \\
10 & 56413933 & 37583 & 5.47 & Outer membrane protein A & OmpA & 103 \\
11 & 56413728 & 28035 & 5.51 & Putative outer membrane protein & mipA & 89 \\
12 & 56414068 & 18540 & 5.74 & Outer membrane protein x precursor & OmpX & 113 \\
\hline
\end{tabular}

were identified as the same three proteins and one weakly stained spot was not identified. Twelve protein spots identified were located in the outer membrane (Table III).

Immunoprotection from the recombinant outer membrane proteins (active immunity). BALB/c mice were vaccinated with the purified recombinant proteins. When the antibody titers were $>1: 1,000$, an intraperitoneal challenge experiment was conducted on the vaccinated mice using CMCC 50973. The preliminary results revealed that strong immunoprotection was provided by LamB, pagC, TolC, nmpC and fadL, with protection rates of $95,95,85,80$ and $70 \%$, respectively. A statistically significant difference $(\mathrm{F}=51.536, \mathrm{P}<0.05$; twosided) was observed between the experimental group and the control group (10\% protection rate). Low protection rates were exhibited by btuB, OmpX and mipA of 50, 40 and $35 \%$, respectively. A significant difference between the experimental group and the control group $(\mathrm{F}=7.77, \mathrm{P}<0.05$; two-sided) was observed. No statistical difference between the control group and the ompC, OmpA, ompW and imp experimental groups $(\mathrm{F}=0.568, \mathrm{P}<0.05$; two-sided) was observed (Table IV).
Immunoproteomics of the recombinant outer membrane protein antibodies (passive immunity). One hour after the mice were vaccinated with single antiserum (with 1:1,000 antibody titers) of recombinant protein with immunoprotection (namely LamB, pagC, TolC, nmpC and fadL), a challenge experiment was conducted on the mice by intraperitoneal infection with five times the $\mathrm{LD}_{50}$ of CMCC 50973. The results showed that apart from 1 mouse injected with the nmpC antiserum that died on the third day, no other mice died within 3 days in the experimental groups. The protection rates of the recombinant protein antisera LamB, pagC, TolC, nmpC and fadL within 7 days were $65,55,60,55$ and $50 \%$, respectively. A significant difference was observed between the experimental group and the control group $(\mathrm{F}=15.771, \mathrm{P}<0.05$; two-sided). The experiment was repeated as previously described using a mixture of the same volumes of five antisera instead of a single antiserum. The results showed that 1 mouse died on the sixth day and 2 died on the seventh day; the protection rate was $90 \%$, which was higher than that of any single antiserum. A significant difference $(\mathrm{F}=7.627, \mathrm{P}<0.05$; two-sided) (Table $\mathrm{V}$ ) was observed. 
Table IV. Results of the mice challenge experiment using recombinant outer membrane proteins.

\begin{tabular}{|c|c|c|c|c|c|c|c|c|c|}
\hline & \multirow{2}{*}{$\begin{array}{c}\text { No. of experimental } \\
\text { animals }\end{array}$} & \multicolumn{7}{|c|}{ No. of deaths } & \multirow{2}{*}{$\begin{array}{l}\text { Protection } \\
\text { rate }(\%)\end{array}$} \\
\hline & & 1st day & 2nd day & 3rd day & 4th day & 5th day & 6th day & 7 th day & \\
\hline LamB & 20 & 0 & 0 & 0 & 0 & 0 & 1 & 0 & $95^{\mathrm{a}}$ \\
\hline pagC & 20 & 0 & 0 & 0 & 0 & 1 & 0 & 0 & $95^{\mathrm{a}}$ \\
\hline TolC & 20 & 0 & 0 & 2 & 0 & 0 & 0 & 1 & $85^{\mathrm{a}}$ \\
\hline $\mathrm{nmpC}$ & 20 & 0 & 0 & 2 & 0 & 1 & 0 & 1 & $80^{\mathrm{a}}$ \\
\hline fadL & 20 & 0 & 0 & 1 & 2 & 0 & 2 & 1 & $70^{\mathrm{a}}$ \\
\hline btuB & 20 & 0 & 3 & 1 & 2 & 1 & 2 & 1 & $50^{\mathrm{b}}$ \\
\hline OmpX & 20 & 0 & 2 & 4 & 2 & 3 & 0 & 1 & $40^{\mathrm{b}}$ \\
\hline $\operatorname{mipA}$ & 20 & 0 & 2 & 3 & 3 & 2 & 1 & 2 & $35^{\mathrm{b}}$ \\
\hline ompC & 20 & 2 & 2 & 4 & 3 & 4 & 1 & 1 & $15^{\mathrm{c}}$ \\
\hline OmpA & 20 & 3 & 3 & 1 & 4 & 3 & 1 & 2 & $15^{\mathrm{c}}$ \\
\hline ompW & 20 & 3 & 2 & 3 & 4 & 3 & 1 & 2 & $10^{\mathrm{c}}$ \\
\hline $\mathrm{imp}$ & 20 & 3 & 2 & 4 & 3 & 4 & 1 & 1 & $10^{c}$ \\
\hline Control & 20 & 1 & 3 & 6 & 3 & 4 & 1 & 0 & 10 \\
\hline
\end{tabular}

${ }^{a} \mathrm{LamB}, \operatorname{pagC}, \mathrm{TolC}, \mathrm{nmpC}$ and fadL exhibited good immunoprotection, with protection rates at 95, 95, 85, 80 and 70\%, respectively. A statistically significant difference $(\mathrm{F}=51.536, \mathrm{P}<0.05$; two-sided) was observed between the experimental group and the control group (10\% protection

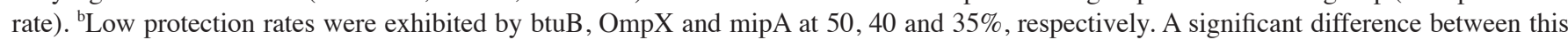
experimental group and the control group $(\mathrm{F}=7.77, \mathrm{P}<0.05$; two-sided $)$ was observed. ${ }^{\mathrm{c}}$ No statistical difference between the control group and the ompC, OmpA, ompW and imp experimental group $(\mathrm{F}=0.568, \mathrm{P}<0.05$; two-sided $)$ was observed.

Table V. Immunoprotection from recombinant outer membrane protein antisera.

\begin{tabular}{|c|c|c|c|c|c|c|c|c|c|}
\hline & \multirow{2}{*}{$\begin{array}{c}\text { No. of experimental } \\
\text { animals }\end{array}$} & \multicolumn{7}{|c|}{ No. of deaths } & \multirow{2}{*}{$\begin{array}{l}\text { Protection } \\
\text { rate }(\%)\end{array}$} \\
\hline & & 1st day & 2nd day & 3rd day & 4th day & 5 th day & 6th day & 7th day & \\
\hline LamB & 20 & 0 & 0 & 0 & 2 & 1 & 2 & 2 & $65^{\mathrm{a}}$ \\
\hline pagC & 20 & 0 & 0 & 0 & 1 & 2 & 3 & 3 & $55^{\mathrm{a}}$ \\
\hline TolC & 20 & 0 & 0 & 0 & 1 & 2 & 2 & 3 & $60^{\mathrm{a}}$ \\
\hline $\mathrm{nmpC}$ & 20 & 0 & 0 & 1 & 2 & 2 & 1 & 3 & $55^{\mathrm{a}}$ \\
\hline fadL & 20 & 0 & 0 & 0 & 1 & 2 & 4 & 3 & $50^{\mathrm{a}}$ \\
\hline Mixed sera & 20 & 0 & 0 & 0 & 0 & 1 & 0 & 1 & $90^{\mathrm{b}}$ \\
\hline Control & 20 & 2 & 4 & 5 & 3 & 1 & 1 & 2 & 10 \\
\hline
\end{tabular}

${ }^{\mathrm{a}}$ The protection rates of the recombinant protein antisera LamB, pagC, TolC, nmpC and fadL within 7 days were $65,55,60,55$ and $50 \%$, respectively. A significant difference was observed between the experimental group and the control group $(\mathrm{F}=15.771, \mathrm{P}<0.05$; two-sided $) .{ }^{\mathrm{b}}$ The protection rate of the mixed sera was $90 \%$, which was significantly higher than that of any single antiserum $(\mathrm{F}=7.627, \mathrm{P}<0.05$; two-sided).

\section{Discussion}

The traditional typhoid fever triplex vaccine consists of inactivated Salmonella that causes typhoid fever, paratyphoid fever A and type B paratyphoid. Although it is effective, it provides a short duration of effective immunity and significant side effects. Its adverse reaction is mainly caused by lipopolysaccharides, according to Begier et al (12). Mice vaccinated with O-specific polysaccharide bound to tetanustoxoid demonstrated evident immune response to $S$. paratyphi A (13). The National Institutes of Health used two methods from Sanofi Pasteur to produce a paratyphoid fever
A conjugate vaccine in 2000 , with the clinical observation phases 1 and 2 conducted in Vietnam. Its use was stopped due to its unstable effect and other factors (14). No Vi antigen exists for $S$. paratyphi $A(15,16)$. Therefore, the use of a recombinant protein as the vaccine antigen is a unique and feasible way of developing a new vaccine through genetic engineering.

$S$. paratyphi $A$ is a Gram-negative bacterium without a capsule, whose outer membrane protein plays a key role in pathogenesis and in stimulating the immune response. In the present study, the serum from mice vaccinated with the total outer membrane proteins of CMCC 50973 was capable of 
effectively killing $S$. paratyphi A in vitro. The subsequent challenge experiment performed on the vaccinated mice showed that the $S$. paratyphi $A$ outer membrane proteins conferred immunoprotection and resisted the virulent $S$. paratyphi $A$ infection (the protection rate was $85 \%$ ). The results demonstrated that a number of outer membrane proteins encoded by $S$. paratyphi $A$ are strong protective antigens.

Immunoproteomics is one branch of proteomics that promises to be effective in identifying vaccine targets. Most bacterial outer membrane protein antigens are neutralizing antigens that play a key role in activating the immune system and inducing immunoprotection. The membrane protein, particularly the outer membrane protein, antigens usually have strong immunogenicities, which closely interact with the immune system. Therefore, the surface proteins of pathogens play an essential role in the development of new vaccines $(17,18)$. The use of proteins as bacterial immunogens has many advantages. First, they are highly conserved and can be exposed on the bacterial surface or be secreted by various bacteria. Second, they have strong immunogenicity and induce the serum-independent cross-protective immune response. In addition, they activate the $\mathrm{T}$ cell-dependent immune response and produce memory cells to produce a long-term immune effect. Progress has been made in the research of bacterial protein vaccines, such as Vibrio, Helicobacter pylori and Brucella (19-22). In the present study, the outer membrane proteins of $S$. paratyphi A were investigated using immunoproteomic technology. A preliminary screening was carried out and 12 outer membrane proteins that had strong reactions with patient sera were obtained through bioinformatic research projects. The results of the $S$. paratyphi A active immunity challenge experiment demonstrated that the recombinant proteins Lamb, pagC, TolC, nmpC and fadL provide good immunoprotection. This was supported by the results of the passive immunity challenge experiment using recombinant protein antiserum. The protection rate was close to $100 \%$ within 3 days and the mice began to die gradually on the fourth day. The decline in the protection rates was probably caused by the decrease in antibodies. The immunoprotective rate of the five-antiserum combination was boosted immunoprotection, suggesting that the mixed antiserum had a synergistic effect.

In summary, the LamB, pagC, TolC, nmpC and fadL outer membrane proteins of $S$. paratyphi $A$ have strong synergistic immunogenicity and immunoprotection. An in-depth study of the optimal combination of these recombinant proteins must be conducted in order to design a protein target for a new paratyphoid vaccine. The safety of the proteins must also be investigated. These studies may provide a basis for the development of protein vaccines for $S$. paratyphi $A$.

\section{Acknowledgements}

This study was approved by the Ethics Committees of the Medical College of Xiamen University. It was supported by the National Natural Science Foundation's Major Research Planning (grant no. 91029729), the Natural Science Foundation of Fujian Province (grant no. 2009J01200), the key projects in the Fujian Province Science and Technology Program (grant no. 2009D019).

\section{References}

1. Woods CW, Murdoch DR, Zimmerman MD, et al: Emergence of Salmonella enterica serotype paratyphi A as a major cause of enteric fever in Kathmandu, Nepal. T Roy Soc Trop Med H 100: 1063-1067, 2006

2. Ochiai RL, Wang XY, von Seidlein L, et al: Salmonella paratyphi A rates, Asia. Emerg Infect Dis 11: 1764-1766, 2005.

3. Imran ZP, Zaidi A, Siddiqui AA, Nair S and Ahmad A: Molecular and epidemiological analysis of multidrug-resistant Salmonella paratyphi A obtained from different regions of Pakistan. Pak J Bot 42: 2941-2947, 2010.

4. Crump JA and Mintz ED: Global trends in typhoid and paratyphoid fever. Clin Infect Dis 50: 241-246, 2010.

5. Khanal B, Sharma SK, Amatya R and Poudyal N: Salmonella Typhi and Paratyphi A in Eastern Nepal-trends in antimicrobial resistance. Int J Infect Dis 12: e107-e109, 2008.

6. Uto T, Tsujimura K, Uchijima M, et al: A novel vaccine strategy to induce mycobacterial antigen-specific Th1 responses by utilizing the C-terminal domain of heat shock protein 70. Fems Immunol Med Mic 61: 189-196, 2011.

7. Serpa JA, Valayam J, Musher DM, Rossen RD, Pirofski LA and Rodriguez-Barradas MC: $\mathrm{V}(\mathrm{H}) 3$ antibody response to immunization with pneumococcal polysaccharide vaccine in middle-aged and elderly persons. Clin Vaccine Immunol 18: 362-366, 2011.

8. Wang JC, Ouyang YS, Wang SJ, et al: Construction and expression of nucleic acid vaccine pVAX1-h-alpha S1-140 coding human alpha-synuclein. Neural Regen Res 3: 1372-1375, 2008.

9. Findlow J, Borrow R, Snape MD, et al: Multicenter, open-label, randomized phase II controlled trial of an investigational recombinant meningococcal serogroup $\mathrm{B}$ vaccine with and without outer membrane vesicles, administered in infancy. Clin Infect Dis 51: 1127, 2010.

10. Wooldridge KG and Williams PH: Sensitivity of Escherichia coli to cloacin DF13 involves the major outer membrane protein OmpF. J Bacteriol 173: 2420-2424, 1991.

11. Li H, Xiong XP, Peng B, et al: Identification of broad crossprotective immunogens using heterogeneous antiserum-based immunoproteomic approach. J Proteome Res 8: 4342-4349, 2009.

12. Begier EM, Burwen DR, Haber P, Ball R and Vaccine Adverse Event Reporting System Working Group: Postmarketing safety surveillance for typhoid fever vaccines from the Vaccine Adverse Event Reporting System, July 1990 through June 2002. Clin Infect Dis 38: 771-779, 2004.

13. Konadu E, Shiloach J, Bryla DA, Robbins JB and Szu SC: Synthesis, characterization, and immunological properties in mice of conjugates composed of detoxified lipopolysaccharide of Salmonella paratyphi A bound to tetanus toxoid with emphasis on the role of O acetyls. Infect Immun 64: 2709-2715, 1996.

14. Konadu EY, Lin FY, Ho VA, et al: Phase 1 and phase 2 studies of Salmonella enterica serovar paratyphi A O-specific polysaccharide-tetanus toxoid conjugates in adults, teenagers, and 2- to 4-year-old children in Vietnam. Infect Immun 68: 1529-1534, 2000

15. Yang $\mathrm{HH}, \mathrm{Wu} \mathrm{CG}, \mathrm{Xie} \mathrm{GZ}$, et al: Efficacy trial of Vi polysaccharide vaccine against typhoid fever in south-western China. Bull World Health Organ 79: 625-631, 2001.

16. Parry $\mathrm{C}$, Hien $\mathrm{T}$ and Dougan G: Review article: typhoid fever. N Engl J Med 347: 1770-1782, 2002

17. Sinha S, Kosalai K, Arora S, et al: Immunogenic membraneassociated proteins of Mycobacterium tuberculosis revealed by proteomics. Microbiology 151: 2411-2419, 2005.

18. Maione D, Margarit I, Rinaudo CD, et al: Identification of a universal Group B streptococcus vaccine by multiple genome screen. Science 309: 148-150, 2005.

19. Li NQ, Bai JJ, Wu SQ, et al: An outer membrane protein, OmpK, is an effective vaccine candidate for Vibrio harveyi in Orange-spotted grouper (Epinephelus coioides). Fish Shellfish Immun 25: 829-833, 2008.

20. Talebkhan Y, Mohammadi M, Razavi N and Sadeghi M: Overexpression of outer membrane protein $18, \mathrm{Omp} 18$, as a putative vaccine against $H$-pylori infection. Helicobacter 13: 468-469, 2008.

21. Qian RH, Xiao ZH, Zhang CW, et al: A conserved outer membrane protein as an effective vaccine candidate from Vibrio alginolyticus. Aquaculture 278: 5-9, 2008.

22. Gupta VK, Rout PK and Vihan VS: Induction of immune response in mice with a DNA vaccine encoding outer membrane protein (omp31) of Brucella melitensis 16M. Res Vet Sci 82: 305-313, 2007. 